\title{
Making the most of Pap tests
}

The paper by Taylor and colleagues ${ }^{1}$ in this issue of the journal indicates that in NSW, (a state of Australia with a population of approximately 2.4 million adult women ${ }^{2}$ ), cervical cancer screening prevented the development of around 3400 cases of cervical cancer between 1972 and 1996. Projections of the population, cervical cancer incidence and effectiveness of screening to 2006 suggest that close to 8000 cases of cervical cancer will be prevented by 2006 . Their analysis indicates that in the interval 1972-1996, 1200-1600 deaths from cervical cancer were averted.

As Taylor et al note there are no randomised trials of cervical cancer screening so individuals and organisations wishing to quantify the effectiveness of screening are forced to rely on observational data examining trends in incidence and mortality in relation to the introduction and intensity of screening programmes. Recent papers ${ }^{3-5}$ are consistent with Taylor et al in demonstrating an impact of screening over the past decade and add to the body of observational literature indicating that cervical cancer screening does indeed work. For example, using mortality data from England and Wales, Sasieni and Adams estimate that there were about 8000 fewer cervical cancer deaths between 1988 and 1997 as a result of screening. ${ }^{3}$

Cervical cancer screening has made an important impact on women's health. But that is not to say that it could not be improved. Indeed one of the assumptions of Taylor et al in their modelling of the predicted benefits of screening into the future is that "there are continued improvements in cervical screening and its consequences."

So what can be done to improve cervical cancer screening? One of the drawbacks of cervical screening is the relatively low sensitivity and specificity of the Pap test. A recent systematic review that examined the accuracy of conventional manual screening reported that based on the 12 studies with the "least biased estimates sensitivity ranged from $30 \%$ to $87 \%$ and specificity ranged from $86 \%$ to $100 \% " .{ }^{6}$ While a specificity of $86 \%$ to $100 \%$ may sound impressive, this is in the context of a screening programme that screens literally millions of women for a rare disease. Even a specificity of $95 \%$ will mean that for every 100000 women who do not have cervical cancer or high grade cervical abnormalities, 5000 women will have a false positive result. This is an unintended and unwanted side effect of the cervical screening programme. As Raffle ${ }^{7}$ put it "Who would have thought that we would be diagnosing abnormal smear tests in 6800 of every 100000 women screened, when the annual incidence of invasive cervical cancer in England and Wales was never greater than 30 per 100000 ?"

Lack of sensitivity is also a problem with the Pap test. Cytologists must screen in the order of 200 000-300 000 cells on every slide and consequently abnormalities, even with the highest quality control procedures in place, are missed. These "false negatives" are an inevitability in current cervical screening programmes but this has not stopped successful claims for compensation for missed cancers. $^{8}$ Better understanding by lawyers and judges might help, but the fact remains that the majority of people currently think that compensation is warranted for missed cancers. ${ }^{9}$ An alternative is to have a better test.

There are a range of new approaches to cervical screening currently being evaluated including monolayer smear techniques and computer assisted cytology. ${ }^{10}$ For example, the PRISMATIC trial is a high quality evaluation of PAPNET assisted primary screening. ${ }^{11}$ PAPNET is a neural network technology that displays cells on a conventionally prepared Pap smear slide on a high resolution computer screen and flags abnormal cells for cytologists' attention. The cytologist then reviews carefully any slide containing abnormalities. Slides regarded as normal by the computer are screened only briefly by microscope before being signed out. Many studies have demonstrated that PAPNET and other computer assisted techniques can identify additional abnormal slides when applied as a rescreening or quality control procedure. PRISMATIC is one of the first trials to evaluate this approach for primary screening rather than rescreening. The PRISMATIC trial demonstrated that PAPNET assisted primary screening had similar sensitivity $(82 \%)$ to conventional screening $(83 \%)$ with significantly better specificity and in significantly less time than conventional screening. Thus computer assisted approaches to screening have considerable potential to ease the load on cytologists, to free up their time for assessment of difficult slides and to lift the accuracy of cervical screening.

Finally, to return to the subject of unrealistic expectations in the community about the benefits that screening programmes in general can deliver. The General Medical Council has produced guidelines, which require that people considering screening are advised by doctors or other parties about the purpose of screening, the likelihood of negative and positive findings including false negative and false positive results, uncertainties and risks of screening and important medical, social and financial consequences of screening. ${ }^{12}$

The provision of such information is no trivial task yet it clearly can no longer be avoided so how are we going to achieve it? One way is through the development of decision aids that provide people with the information they need to make the decision and with help in achieving each step in the decision making process. Decision aids are defined as "interventions designed to help people make specific and deliberative choices among options by providing ... .information on the disease or condition; probabilities of outcomes ..., an explicit exercise to clarify values; information on others opinions and guidance or coaching in the steps of decision making". ${ }^{13}$ Decision aids are not intended to influence the decision taken but to support informed choice. A number are available for treatment choices but there is a dearth of decision aids available for screening choices yet this may be an area in which decision aids can make an important contribution by helping people make informed choices about whether to participate in screening.

Many will argue that individual decision making in the context of population based screening is too time consuming and unrealistic in a world that is increasingly time poor. Another option is the approach of community informed consent, which advocates that representative individuals go through the process of evidence-based decision making on behalf of an entire community or subset of a community. ${ }^{14}$ In instances where the vast majority elect against screening, policy makers may decide not to implement the programme. Where there is community consensus that screening is considered worthwhile and is reasonably cost effective, the programme is implemented and individual participants can then choose either to go through the process of informed choice themselves or to accept the judgement of the community that screening is worthwhile.

ALEXANDRA BARRATT

Department of Public Health, A27 University of Sydney, Sydney, NSW, Australia 2006 
1 Taylor RJ, Morrell SL, Mamoon HA, et al. Effects of screening on cervical cancer incidence and mortality in New South Wales implied by influences of period of diagnosis and birth cohort. 7 Epidemiol Community Health 2001;55:782-8.

2 Australian Bureau of Statistics, 1999 (Catologue number 3235.1).

3 Sasieni P, Adams J. Effect of screening on cervical cancer mortality in England and Wales: analysis of trends with an age period cohort model. BMF 1999;318:1244-5.

4 Sasieni PD, Cuzick J, Lynch-Farmery E. National Co-ordinating Network for Cervical Screening Working Group. Estimating the efficacy of screening by auditing smear histories of women with and without cervical cancer. $\mathrm{Br}$ f Cancer 1996;73:1001-5.

5 Quinn M, Babb P, Jones J, et al. Effect of screening on incidence of and mortality from cancer of the cervix in England: evaluation based on routinely collected statistics. BMF 1999;318:904-8.

6 Nanda K, McCrory DC, Myers ER, et al. Accuracy of the Papanicolaou test in screening for and follow-up of cervical cytologic abnormalities: a in screening for and follow-up of cervical cytologic

7 Raffle AE. New tests in cervical screening. Lancet 1998;351:297.
8 Dyer C. Three women win in cancer screening case. BMf 1999;318:484.

9 Barratt A, Cockburn J, Furnival C, et al. Perceived sensitivity of mammographic screening: womens' views on test accuracy and financial compensation for missed cancers. F Epidemiol Community Health 1999;53: 716-20.

10 Broadstock M. Effectiveness and cost-effectiveness of automated and semiautomated cervical screening devices. A systematic review of the literature. NZHTA Report 2000; 3(1).

11 PRISMATIC Project Management Team. Assessment of automated primary screening on PAPNET of cervical smears in the PRISMATIC trial. Lancet 1999;353:1381-5.

12 General Medical Council. Seeking patients' consent: the ethical considerations. London: GMC, 1999.

13 O'Connor A, Fiset V, DeGrasse C, et al. Decision aids for patients considering options affecting cancer outcomes: evidence of efficacy and policy implications. Monogr Natl Cancer Inst 1999;25:67-80.

14 Irwig L, Glasziou P. Informed consent for screening by community sampling. Effective Clinical Practice 2000;3:47-50.

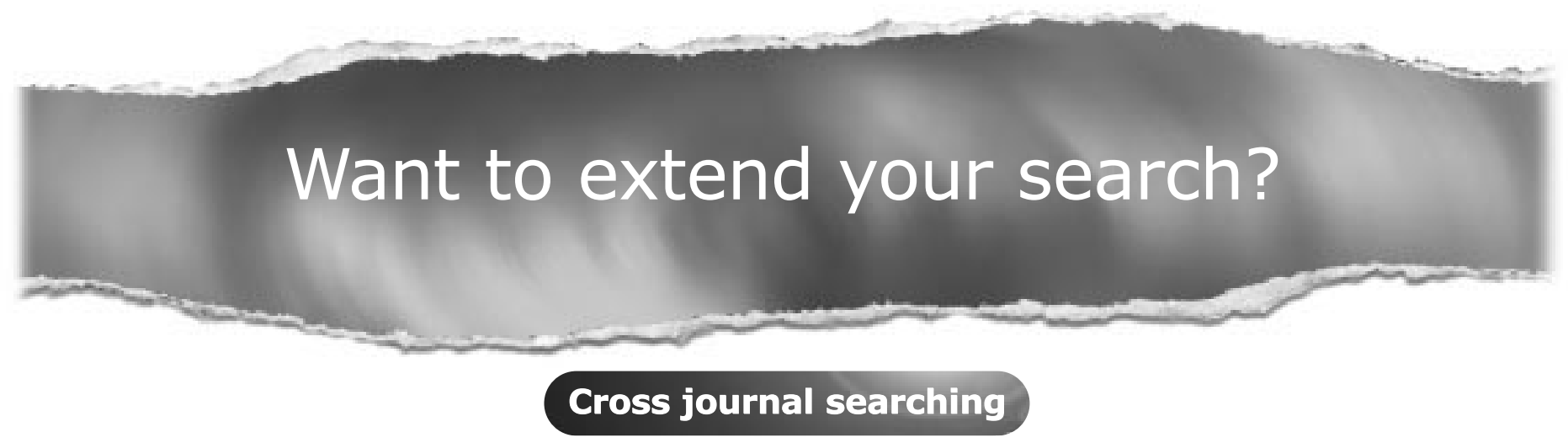

If you can't find what you are looking for in the Journal of Epidemiology and Community Health you can extend your search across many of the more than 200 journals available for selection. You can restrict your search to specific subject areas

(eg, clinical medicine, basic research), or select specific journals, or search all available titles.

www.jech.com 